\title{
Metabolic responses of avocado plants to stress induced by Rosellinia necatrix analysed by fluorescence and thermal imaging
}

Espen Granum, Estación Experimental del Zaidín, Consejo Superior de Investigaciones Científicas, Profesor Albareda 1, 18008 Granada, Spain; María Luisa Pérez-Bueno, Estación Experimental del Zaidín, Consejo Superior de Investigaciones Científicas, Profesor Albareda 1, 18008 Granada, Spain; Claudia E. Calderón, Instituto de Hortofruticultura Subtropical y Mediterránea La Mayora, Universidad de Málaga, Consejo Superior de Investigaciones Científicas, Departamento de Microbiología, Facultad de Ciencias, Campus de Teatinos, 29071 Málaga, Spain; Cayo

Ramos, Instituto de Hortofruticultura Subtropical y Mediterránea La Mayora, Universidad de Málaga, Consejo Superior de Investigaciones Científicas, Área de Genética, Facultad de Ciencias, Campus de Teatinos, 29071 Málaga, Spain; Antonio de Vicente, Instituto de Hortofruticultura Subtropical y Mediterránea La Mayora, Universidad de Málaga, Consejo Superior de Investigaciones Científicas, Departamento de Microbiología, Facultad de Ciencias, Campus de Teatinos, 29071 Málaga, Spain;

Francisco M. Cazorla, Instituto de Hortofruticultura Subtropical y Mediterránea La Mayora, Universidad de Málaga, Consejo Superior de Investigaciones Científicas, Departamento de Microbiología, Facultad de Ciencias, Campus de Teatinos, 29071 Málaga, Spain; Matilde Barón, Estación Experimental del Zaidín, Consejo Superior de Investigaciones Científicas, Profesor Albareda 1, 18008 Granada, Spain 
One of the most important soilborne diseases affecting avocado (Persea americana Mill.) crops is white root rot, caused by the fungus Rosellinia necatrix. In this study we investigated, as a model, the metabolic response elicited by white root rot in the airborne part of the plant as well as the potential applications of imaging techniques, including chlorophyll fluorescence, blue-green fluorescence and thermography, in early detection of white root rot on leaves of avocado plants. The results show that plant metabolism is significantly affected by the infection only when visual symptoms appear, probably related to the loss in functionality of the roots. However, changes in some chlorophyll fluorescence parameters provided early indications of stress related to the fungal infection prior to the development of visual symptoms in the aerial part of the plant. We suggest that the combinatorial analysis of several fluorescence parameters could be used as a method for early detection of biotic and abiotic stress in avocado plants, and in particular of avocado white root rot. 


\section{Introduction}

One of the most important diseases affecting avocado (Persea Americana Mill.) plantations is white root rot, caused by the ascomycete fungus Rosellinia necatrix (Pliego et al. 2009, Pliego et al. 2012, Sztejnberg \& Madar 1980). This disease has caused great economic losses in other woody crops, including ornamental and fruit trees (Pliego et al. 2012). Symptoms of white root rot include rotting of the roots and yellowing of the leaves that eventually wilt. Death of the trees occurs within a few weeks after the appearance of the first foliar symptoms. Efforts have been made over the past three decades to achieve control of $R$. necatrix by different strategies, including the use of fungicides (Kanadani et al. 1998), soil solarisation (López-Herrera et al. 1998), biological control (Calderón et al. 2013, Cazorla et al. 2006, Freeman et al. 1986, Pliego et al. 2007, Pliego et al. 2008) or a combination of the former (Ruano-Rosa et al. 2014). Nevertheless, the economic losses caused by $R$. necatrix in crop fields are increasing worldwide, as reviewed by Pliego et al. (2012).

Imaging techniques applied to remote sensing are powerful tools to be used in crop protection (Mahlein et al. 2012). Analysis of chlorophyll fluorescence is a widely used method in the study of photosynthesis, since it measures indirectly the activity of the electron transport chain of the chloroplast. The photosynthetic apparatus is a primary target of stress, particularly the chloroplast thylakoid membrane. In this membrane, photosystem II catalyses the production of $\mathrm{O}_{2}$ and is the main source of reactive oxygen species that can cause oxidative damage. An important defence mechanism of the chloroplast is the dissipation of energy in a safe form, mainly as heat and fluorescence. Therefore, chlorophyll fluorescence parameters such as the maximum efficiency of photosystem II $\left(\mathrm{F}_{\mathrm{V}} / \mathrm{F}_{\mathrm{M}}\right)$ and non-photochemical quenching (NPQ) can be 
sensitive indicators of plant stress. Indeed, chlorophyll fluorescence imaging has been used in the study of many plant-pathogen interactions (Gorbe \& Calatayud 2012, Rolfe \& Scholes 2010).

Multicolour fluorescence imaging consists of capturing fluorescence images in an assortment of regions of the spectrum to characterize the response of plant secondary metabolism to stress (Bilger et al. 2001, Cerovic et al. 1999). In particular, fluorescence in the blue and green regions is emitted by phenolic compounds with a wide range of roles in plant defence. Hence, this method provides a useful tool to discriminate stressed plants from healthy ones (Buschmann \& Lichtenthaler 1998).

Thermal imaging provides measurements of leaf temperature, which is an indicator of transpiration and stomatal conductance (Chaerle \& Van der Straeten 2000, Glenn 2012). In response to pathogen infection or water deficit, stomatal closure results in restricted transpiration and an increase in leaf temperature.

The aim of this work was to analyse the effects of $R$. necatrix infection in the airborne part of the trees and to investigate the potential applications of the already mentioned imaging techniques in presymptomatic detection of stress in avocado plants, particularlyof avocado white root rot.

\section{Materials and Methods}

\section{Fungal growth and preparation of inoculum}

Virulent $R$. necatrix strain CH53-GFP was grown at $25^{\circ} \mathrm{C}$ on potato dextrose agar (PDA; Difco Laboratories, Detroit, MI) or TPG plates (Calderón et al. 2013, Pliego et al. 2009) and stored in TPG at $4^{\circ} \mathrm{C}$ as previously described (Gutiérrez-Barranquero et al. 2012). 


\section{Plant growth and infection}

Artificial inoculations to reproduce avocado white root rot were performed using the $P$. americana/R. necatrix system as described previously (Cazorla et al. 2006).

Commercial six-month-old avocado plants were obtained from Brokaw nurseries

(Brokaw España, S.L., Vélez-Málaga, Spain). The plants were placed into pots containing $30 \mathrm{~g}$ of wet potting soil (Jongkind Grond B.V., Aalsmeer, The Netherlands) and infested with $R$. necatrix grown on four infected wheat grains per pot as described previously (Freeman et al. 1986). Then, the plants were mantained at 16/8 h light/dark regime at $24 / 18^{\circ} \mathrm{C}, 60 \%$ relative humidity and $200 \mu \mathrm{mol}$ photon $\mathrm{m}^{-2} \mathrm{~s}^{-1}$. The plants were analysed at mid-photophase every 2-3 days throughout the period of study. For each experiment, at least five plants per treatment were analysed. The experiment was repeated six times with similar results.

\section{Chlorophyll fluorescence imaging}

Chlorophyll fluorescence (Chl-F) imaging was carried out with a customised Open FluorCam FC 800-O (PSI, Brno, Czech Republic) kinetic imaging fluorometer controlled by FluorCam7 (PSI) software. Measuring light flashes (10 $\mu \mathrm{s})$ for modulated Chl-F excitation were generated by a pair of red LED panels $\left(\lambda_{\max } \sim 618 \mathrm{~nm}\right)$, and saturating light pulses $\left(1 \mathrm{~s}, \sim 2000 \mu \mathrm{mol} \mathrm{m}^{-2} \mathrm{~s}^{-1}\right)$ and actinic light by a pair of blue LED panels $\left(\lambda_{\max } \sim 455 \mathrm{~nm}\right)$. Chl-F emission kinetics were captured by a charge-coupled device camera with 12-bit and 72 pixels per inch resolution, taking 10 images per second. Reflected radiation was blocked by a far-red filter (RG697, Schott, Mainz, Germany). Measurements of minimum fluorescence $\left(\mathrm{F}_{0}\right)$ on 30 min dark-adapted leaves and maximum fluorescence $\left(\mathrm{F}_{\mathrm{M}}\right)$ after a saturating light pulse were used to determine maximum quantum yield by the formula $F_{V} / F_{M}=\left(F_{M}-F_{0}\right) / F_{M}$. Changes in maximum 
fluorescence during actinic light (400 $\mu \mathrm{mol}$ photon $\left.\mathrm{m}^{-2} \mathrm{~s}^{-1}\right)$ adaptation were followed during a period of 5 min with saturating light pulses applied after $10 \mathrm{~s}, 2.5 \mathrm{~min}$ and 5 min. Measurements of maximum fluorescence in 5 min light-adapted leaves $\left(\mathrm{F}_{\mathrm{M}}{ }_{\mathrm{M}}\right)$ were used to determine steady-state NPQ in the light by NPQ $=\left(\mathrm{F}_{\mathrm{M}}-\mathrm{F}^{\prime}{ }_{\mathrm{M}}\right) / \mathrm{F}^{\prime}{ }_{\mathrm{M}}$. The measuring data were also used to determine other photosynthetic parameters that are potential stress indicators, including $\mathrm{F}_{\mathrm{M}} / \mathrm{F}_{0}, \mathrm{~F}^{\prime}{ }_{\mathrm{V}} / \mathrm{F}^{\prime}{ }_{\mathrm{M}}, \mathrm{F}^{\prime}{ }_{\mathrm{M}} / \mathrm{F}^{\prime}{ }_{0}$ and $\mathrm{F}^{\prime}{ }_{\mathrm{V}} / \mathrm{F}^{\prime}{ }_{0}$ (where $\mathrm{F}^{\prime}{ }_{\mathrm{V}}=$ $\left.\mathrm{F}^{\prime}{ }_{\mathrm{M}}-\mathrm{F}^{\prime}{ }_{0}\right)$.

\section{Blue-green fluorescence imaging}

Blue-green fluorescence images were captured by the same imaging system using UV (360 nm) excitation light. Fluorescence images in the blue (F440) and green (F520) regions of the spectrum were acquired sequentially using single-band bandpass filters of 442/46 nm and 525/39 nm (Semrock, Illinois, USA), respectively (Pérez-Bueno et al. 2014). The acquisition of each fluorescence image required the accumulation of 9 images captured during $18 \mathrm{~s}$, generating images of $740 \times 480$ pixels with a resolution of 96 pixels per inch.

\section{Thermal imaging}

Infrared images of plant leaves were recorded in the growth chamber with a Photon 640 camera (FLIR Systems Wilsonville, USA) vertically positioned approximately $0.5 \mathrm{~m}$ above the leaves. The Photon 640 is a long-wavelength $(7.5-13.5 \mu \mathrm{m})$ uncooled microbolometer camera with a resolution of $640 \times 512$ pixels $(25 \mu \mathrm{m})$ and NEdT performance $<50 \mathrm{mK}$ at $\mathrm{f} / 1.0$. Digital video data (14-bit serial LVDS) were transmitted through an ethernet adapter to a computer and calibrated by FluorCam 7 software (Photon Systems Instruments). Infected plants and control plants were positioned for 
simultaneous imaging of one leaf from each plant, and the images were used to determine differences in average leaf temperature between treatments $\left(\Delta T=T_{R}-T_{C}\right.$, where $T_{R}$ is the temperature of the infected plant leaf and $T_{C}$ is the temperature of the control plant leaf).

\section{Results}

\section{Development of the fungal infection in plants}

Initial symptoms of fungal infection appeared at 28 days post-inoculation (dpi) in the form of wilt, similar to symptoms of severe water stress (Fig. 1). A few days later (3236 dpi), plant leaves were desiccated and dead.

\section{Effects of fungal infection on host photosynthesis}

Effects of the infection on photosynthetic parameters such as $\mathrm{F}_{\mathrm{V}} / \mathrm{F}_{\mathrm{M}}$ and NPQ were analysed by chlorophyll fluorescence imaging (Fig. 2). $\mathrm{F}_{\mathrm{V}} / \mathrm{F}_{\mathrm{M}}$ remained constant at 0.78 in the leaves of infected plants until the late presymptomatic stage ( $25 \mathrm{dpi}$ ) when $\mathrm{F}_{\mathrm{V}} / \mathrm{F}_{\mathrm{M}}$ was slightly decreased (0.74). When the first symptoms appeared $F_{V} / F_{M}$ decreased dramatically, declining by $50 \%$ at 28 dpi. NPQ remained constant at 1.7 in the leaves of infected plants, and then decreased dramatically when the first symptoms appeared, declining by $75 \%$ at $28 \mathrm{dpi}$.

Some other chlorophyll fluorescence parameters that are potential stress indicators, including $F_{\mathrm{M}} / \mathrm{F}_{0}, \mathrm{~F}^{\prime}{ }_{\mathrm{V}} / \mathrm{F}^{\prime}{ }_{\mathrm{M}}, \mathrm{F}^{\prime}{ }_{\mathrm{M}} / \mathrm{F}^{\prime}{ }_{0}$ and $\mathrm{F}^{\prime}{ }_{\mathrm{V}} / \mathrm{F}^{\prime}{ }_{0}$, were assessed in leaves at 25 and 28 dpi, shortly before and after the appearance of symptoms (Fig. 3). The parameters $F_{M} / F_{0}, F^{\prime}{ }_{V} / F^{\prime}{ }_{M}$ and $F^{\prime}{ }_{V} / F^{\prime}{ }_{0}$ showed significant declines in the leaves of infected plants at the presymptomatic stage ( $25 \mathrm{dpi})$, and further decreased when symptoms appeared (28 dpi). The remaining parameter $\mathrm{F}^{\prime}{ }_{\mathrm{M}} / \mathrm{F}^{\prime}{ }_{0}$ only showed a 
significant decrease in the leaves of infected plants after symptoms appeared (28 dpi).

\section{Effects of fungal infection on host secondary metabolism}

Effects of the infection on host secondary metabolism were assessed by blue-green fluorescence imaging (Fig. 4). A significant increase in blue $\left(\mathrm{F}_{440}\right)$ and green $\left(\mathrm{F}_{520}\right)$ fluorescence in the leaves of infected plants were only observed at the late-symptomatic stage (32-36 dpi), which is probably caused by an alteration in the optical properties of the leaves due to desiccation (Cerovic et al. 1999).

\section{Effects of fungal infection on host transpiration}

Effects of the infection on host transpiration were assessed by thermal imaging (Fig. 5). A significant increase in the leaf temperature $\left(0.5-1.0^{\circ} \mathrm{C}\right)$ of infected plants was recorded at the early to late-symptomatic stage (28-32 dpi), indicating stomatal closure in response to the infection.

\section{Discussion}

$R$. necatrix is a filamentous ascomycete causing white root rot in a wide range of perennial plants (Pliego et al. 2012). When mycelial inoculum of the dormancy stage encounters roots, mycelial masses and strands start to propagate on the roots, penetrate from the lenticels of epidermal cells and junctions between epidermal cells, and then invade the epidermis and xylem of the roots, forming fan-shaped mycelial strands (Pliego et al. 2009). Symptoms in the aerial parts of the plants cannot be recognized in early stages of root infection and, to our best knowledge, there is no study describing early detection of disease effects on leaves. This system was used as a model to explore the applicability of imaging techniques for the detection of metabolic stress on avocado 
leaves.

Root rot causes water stress in the airborne part of the plant. Quite unexpectedly, the photosynthetic parameters Fv/Fm and NPQ, the autofluorescence in the blue and green regions, and the stomatal conductance, which otherwise are good stress indicators (Buschmann \& Lichtenthaler 1998, Mahlein et al. 2012, Rolfe \& Scholes 2010), showed no differences between infected and healthy plants. Therefore, it can be concluded that white root rot causes little immediate impact on metabolism in the leaves since no alterations in photosynthesis, secondary metabolism or transpiration were found prior to the development of visual symptoms. This provides an explanation why, up to date, the only methods available for early detection of the disease are based on direct observation of infected roots or by molecular tools (Kondo et al. 2013), therefore, complicated and laborious.

Only a discrete decrease in the maximum quantum yield of photosystem II could be detected (Fig. 2), soon before the first visual symptoms appear. The decrease in this parameter suggests an oxidative damage to photosystem II, probably due to the water stress caused by the white root rot. However, this inhibition only appeared after 28 dpi, soon before the first symptoms appeared. On the contrary, other chlorophyll fluorescence parameters $\left(\mathrm{F}_{\mathrm{M}} / \mathrm{F}_{0}, \mathrm{~F}^{\prime}{ }_{\mathrm{V}} / \mathrm{F}^{\prime}{ }_{\mathrm{M}}\right.$ and $\left.\mathrm{F}^{\prime}{ }_{\mathrm{V}} / \mathrm{F}^{\prime}{ }_{0}\right)$, offered statistically significant differences between healthy and stressed plants at an earlier stage of the infection. Chlorophyll fluorescence imaging is an inexpensive, non-destructive tool widely used in the study of plant stress. Based on the findings here reported, we suggest that the combined analysis of parameters that offer differences $\left(\mathrm{F}_{\mathrm{M}} / \mathrm{F}_{0}, \mathrm{~F}^{\prime}{ }_{\mathrm{V}} / \mathrm{F}^{\prime}{ }_{\mathrm{M}}\right.$ and $\left.\mathrm{F}^{\prime}{ }_{\mathrm{V}} / \mathrm{F}^{\prime}{ }_{0}\right)$ together with the parameters that should not show differences (the rest of parameters analysed in this study) could be used as disease signatures for early detection of stressed avocado plants potentially infected by $R$. necatrix, but not specifically. This method 
could be used to screen for potentially infected or resistant plants, both in orchards and nurseries, prior to a diagnosis by conventional and more expensive methods of detection of $R$. necatrix. Additionally, these techniques could be also useful as an objective method for quantitative analysis of experimental infections or in the detection of a biotic and abiotic stress conditions on avocado plants.

\section{Acknowledgments}

This work was supported by grants from CICE-Junta de Andalucía, Proyectos de Excelencia (P08-CVI-03475, P10-AGR-5797 and P12-AGR-0370) and Plan Nacional de I+D+I del Ministerio de Ciencia e Innovación, Spain (AGL2011-30354C0201) cofinanced by FEDER, EU. E. Granum was recipient of a JAE-Doc contract funded by CSIC, Spain. C. E. Calderón was supported by a grant from FPI, Ministerio de Ciencia e Innovación, Spain.

\section{References}

Bilger, W., Johnsen, T. \& Schreiber, U. (2001). UV-excited chlorophyll fluorescence as a tool for the assessment of UV-protection by the epidermis of plants. Journal of Experimental Botany, 52(363), 2007-2014.

Buschmann, C. \& Lichtenthaler, H. K. (1998). Principles and characteristics of multi-colour fluorescence imaging of plants. Journal of Plant Physiology, 152(2-3), 297-314.

Calderón, C. E., Pérez-García, A., de Vicente, A. \& Cazorla, F. M. (2013). The dar genes of Pseudomonas chlororaphis PCL1606 are crucial for biocontrol activity via production of the antifungal compound 2-Hexyl, 5-propyl resorcinol. Molecular Plant-Microbe Interactions, 26(5), 554-565.

Cazorla, F. M., Duckett, S. B., Bergström, E. T., Noreen, S., Odijk, R., Lugtenberg, B. J. J., et al. 
(2006). Biocontrol of Avocado Dematophora Root Rot by Antagonistic Pseudomonas fluorescens PCL1606 Correlates With the Production of 2-Hexyl 5-Propyl Resorcinol. Molecular Plant-Microbe Interactions, 19(4), 418-428.

Cerovic, Z. G., Samson, G., Morales, F., Tremblay, N. \& Moya, I. (1999). Ultraviolet-induced fluorescence for plant monitoring: present state and prospects. Agronomie, 19(7), 543-578.

Chaerle, L. \& Van der Straeten, D. (2000). Imaging techniques and the early detection of plant stress. Trends in Plant Science, 5(11), 495-501.

Freeman, S., Sztejnberg, A. \& Chet, I. (1986). Evaluation ofTrichoderma as a biocontrol agent forRosellinia necatrix. Plant and Soil, 94(2), 163-170.

Glenn, D. M. (2012). Infrared and chlorophyll fluorescence imaging methods for stress evaluation. HortScience, 47(6), 697-698.

Gorbe, E. \& Calatayud, A. (2012). Applications of chlorophyll fluorescence imaging technique in horticultural research: A review. Scientia Horticulturae, 138, 24-35.

Gutiérrez-Barranquero, J. A., Pliego, C., Bonilla, N., Calderón, C. E., Pérez-García, A., de Vicente, A., et al. (2012). Sclerotization as a long-term preservation method for Rosellinia necatrix strains. Mycoscience, 53(6), 460-465.

Kanadani, G., Date, H. \& Nasu, H. (1998). Effect of fluazinam soil-drench on white root rot of grapevine. Annals of the Phytopathological Society of Japan, 64(2), 139-141.

López-Herrera, C. J., Pérez-Jiménez, R. M., Zea-Bonilla, T., Basallote-Ureba, M. J. \& MeleroVara, J. M. (1998). Soil Solarization in Established Avocado Trees for Control of Dematophora necatrix. Plant disease, 82(10), 1088-1092.

Mahlein, A. K., Oerke, E. C., Steiner, U. \& Dehne, H. W. (2012). Recent advances in sensing plant diseases for precision crop protection. European Journal of Plant Pathology, 133(1), 197209.

Pérez-Bueno, M. L., Pineda, M., Díaz-Casado, M. E. \& Barón, M. (2014). Spatial and temporal dynamics of primary and secondary metabolism in Phaseolus vulgaris challenged by 
Pseudomonas syringae. Physiologia Plantarum, In Press.

Pliego, C., Cazorla, F. M., Gonzalez-Sanchez, M. A., Perez-Jimenez, R. M., de Vicente, A. \& Ramos, C. (2007). Selection for biocontrol bacteria antagonistic toward Rosellinia necatrix by enrichment of competitive avocado root tip colonizers. Res Microbiol, 158(5), 463-70.

Pliego, C., de Weert, S., Lamers, G., de Vicente, A., Bloemberg, G., Cazorla, F. M., et al. (2008). Two similar enhanced root-colonizing Pseudomonas strains differ largely in their colonization strategies of avocado roots and Rosellinia necatrix hyphae. Environ Microbiol, 10(12), 3295304.

Pliego, C., Kanematsu, S., Ruano-Rosa, D., de Vicente, A., Lopez-Herrera, C., Cazorla, F. M., et al. (2009). GFP sheds light on the infection process of avocado roots by Rosellinia necatrix. Fungal Genet Biol, 46(2), 137-45.

Pliego, C., Lopez-Herrera, C., Ramos, C. \& Cazorla, F. M. (2012). Developing tools to unravel the biological secrets of Rosellinia necatrix, an emergent threat to woody crops. Molecular Plant Pathology, 13(3), 226-39.

Rolfe, S. A. \& Scholes, J. D. (2010). Chlorophyll fluorescence imaging of plant-pathogen interactions. Protoplasma, 247(3-4), 163-75.

Ruano-Rosa, D., Cazorla, F. M., Bonilla, N., Martín-Pérez, R., Vicente, A. \& López-Herrera, C. J. (2014). Biological control of avocado white root rot with combined applications of Trichoderma spp. and rhizobacteria. European Journal of Plant Pathology, 138(4), 751-762.

Sztejnberg, A. \& Madar, Z. (1980). Host range of Dematophora necatrix, the cause of white root rot disease in fruit trees. Plant Disease, 64(7), 662-664. 
Figure legends

Fig. 1. Representative images of $R$. necatrix-infected avocado plant (R) and noninfected control plant (C) at 25 dpi (presymptomatic stage), 28 dpi (early symptoms) and 32 dpi (late symptoms).

Fig. 2. Images and average values of $F_{V} / F_{M}$ (panel $A$ and $B$, respectively) and NPQ (panel C and D, respectively) in leaves of $R$. necatrix-infected avocado plant (R) and non-infected control plant (C) at 25 dpi (presymptomatic stage) and 28 dpi (early symptoms); means \pm sd, $\mathrm{n}=5$.

Fig. 3. Chlorophyll fluorescence parameters $F_{M} / F_{0}$ (panel $\left.A\right), F^{\prime}{ }_{M} / F^{\prime}{ }_{0}($ panel $B), F^{\prime}{ }_{V} / F{ }_{M}$ (panel C) and $\mathrm{F}^{\prime}{ }_{\mathrm{v}} / \mathrm{F}_{0}{ }_{0}$ (panel D) in leaves of $R$. necatrix-infected avocado plant $(\mathrm{R})$ and non-infected control plant (C) at 25 dpi (presymptomatic stage) and 28 dpi (early symptoms); means \pm sd, $\mathrm{n}=5$.

Fig. 4. Blue (F440) fluorescence images and corresponding average values (panel A and $\mathrm{B}$, respectively); and green (F520) fluorescence images and corresponding average values (panel $\mathrm{C}$ and $\mathrm{D}$, respectively). Images of leaves of $R$. necatrix-infected avocado plant (R) and non-infected control plant (C) at 25 dpi (presymptomatic stage) and 28 dpi (early symptoms). means \pm sd, $n=5$.

Fig. 5. Thermal images of leaves of R. necatrix-infected avocado plant (R) and noninfected control plant (C) at 25 dpi (presymptomatic stage) and 28 dpi (early symptoms) (panel A), and difference in average leaf temperature between treatments $(\Delta \mathrm{T})$ throughout the infection (panel B); means \pm sd, $n=5$. 
Click here to download high resolution image
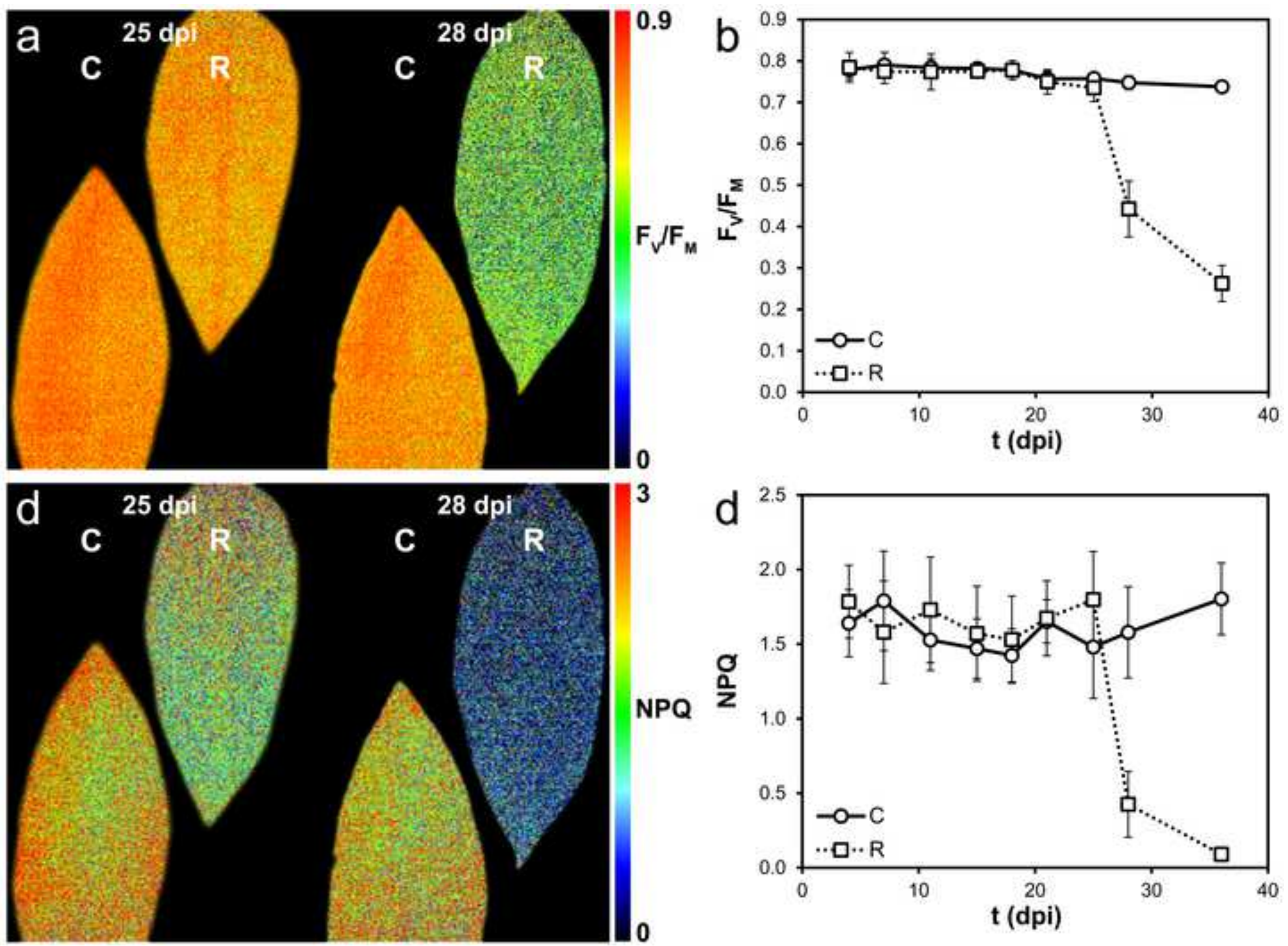

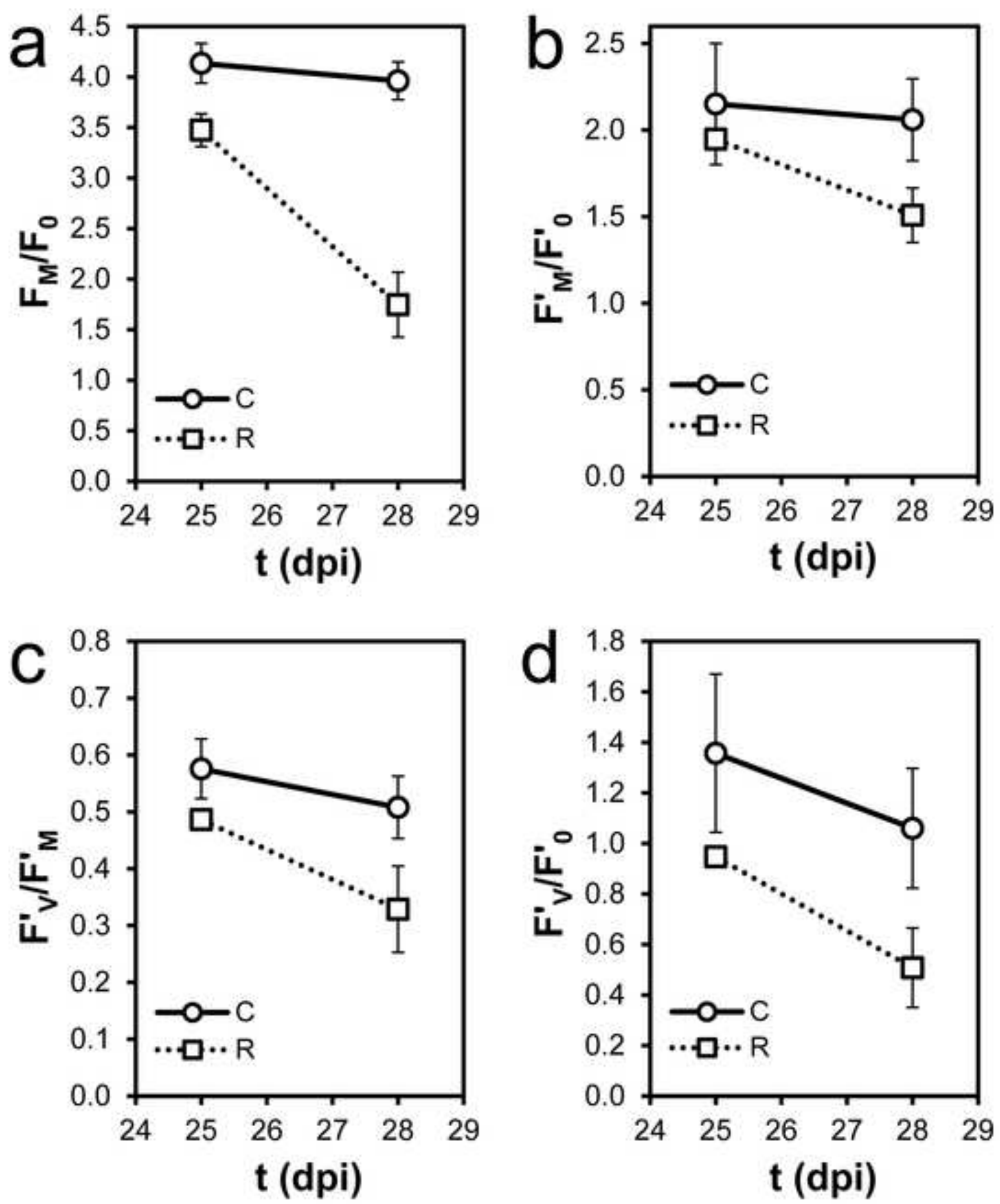

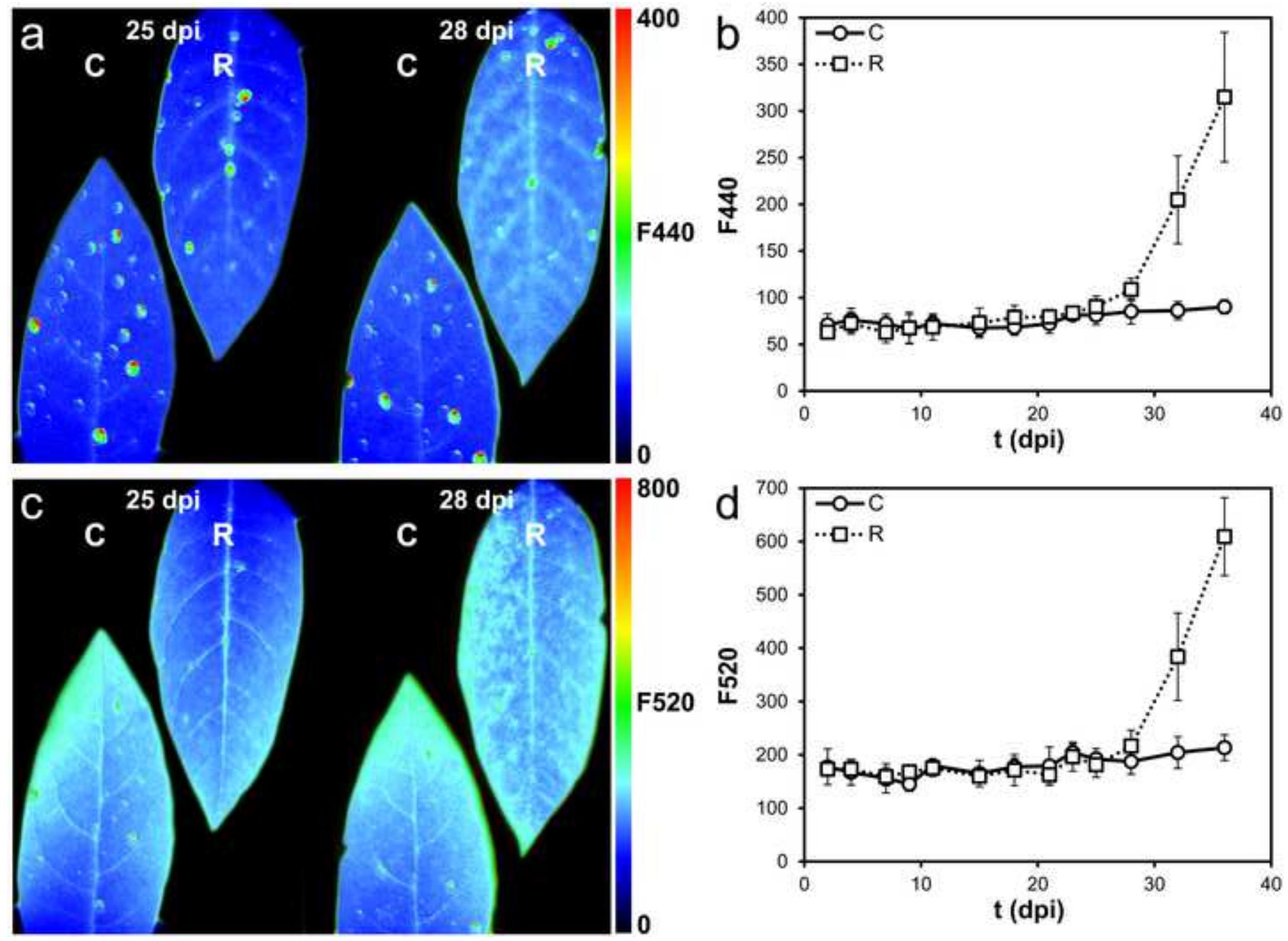


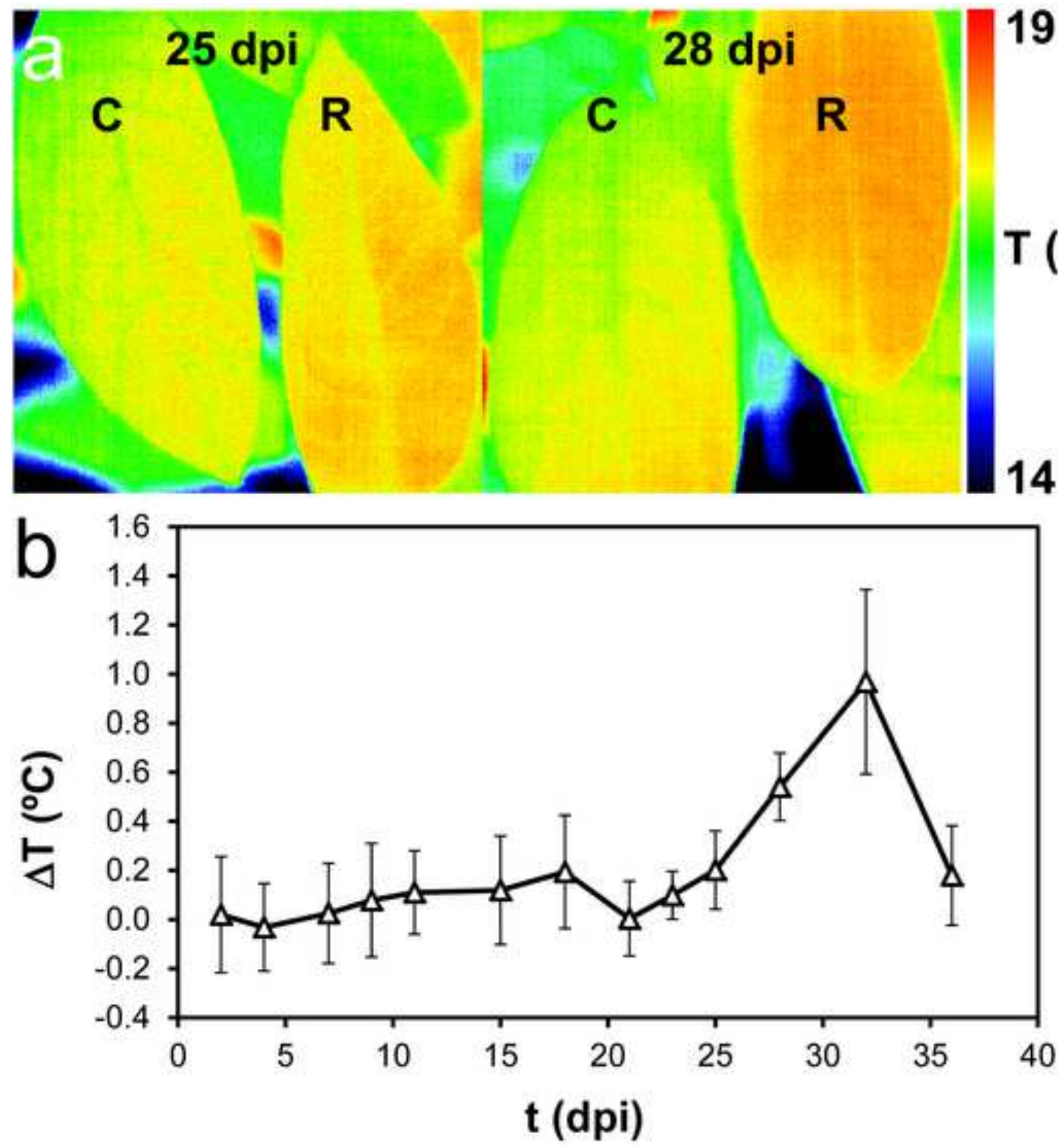

